\title{
Ergebnisse der Tieftemperaturforschung III. Elektronenwärme des Palladiums
}

\author{
Von Klaus Clusius und Liselotte Schachinger \\ Aus dem Physikalisch-Chemischen Institut der Universität München
}

(Z. Naturforschg. 2a, 90-97 [1947]; eingegangen am 5. Dezember 1946)

\begin{abstract}
Die Atomwärme eines Metalls bei konstantem Druck $C_{p}$ besteht gewöhnlich aus vier Anteilen: 1. einem von den Gitterschwingungen herrührenden Betrag $C_{g}$, 2. der Differenz $C_{p}-C_{v}$, 3. der Elektronenwärme $C_{\boldsymbol{e}}$, die nach $\mathrm{S}$ o $\mathrm{mm}$ e r f eld bei freien Elektronen linear mit $T$ ansteigt und 4. einem von $\mathrm{B}$ or $\mathrm{n}$ - B r od y angegebenen Korrekturglied für die Anharmonizität der Gitterschwingungen. Bisher ist die Elektronenwärme überzeugend nur bei tiefen Temperaturen nachgewiesen worden, bei denen die Beiträge 2. und 4. verschwinden; die Gründe für die bei hohen Temperaturen auftretenden Schwierigkeiten eines Nachweises werden diskutiert. Bei stark paramagnetischen und ferromagnetischen Metallen zeigt zwar die Atomwärme bei Heliumtemperaturen ebenfalls ein in $T$ lineares Glied, das einer zusätzlichen Elektronenwärme entspricht, diese ist jedoch rund 10-mal größer, als man für freie Elektronen erwarten muß. Bei diesen Mietallen, z. B. dem Palladium, läßt sich zeigen, daß das einfache $T$-Gesetz für die Elektronenwärme nicht mehr bei hohen Temperaturen gelten kann. Um das in einem größeren Temperaturbereich gültige Gesetz aufzufinden, werden Messungen der wahren Atomwärme des $\mathrm{Pd}$ zwischen 12 und $270^{\circ}$ abs. vorgenommen. Die erhaltenen $C_{p}$. Werte werden auf $C_{v}$ reduziert und diese analysiert. Dabei stellt sich heraus, daß die Elektronenwärme proportional $\chi_{e} T$ verläuft, wobei $\chi_{e}$ die temperaturabhängige paramagnetische Atomsuszeptibilität des Palladiums bedeutet; man erhält
\end{abstract}

$$
C_{e}=2,22 \chi_{e} T .
$$

Der Restbetrag $C_{v}-C_{\boldsymbol{e}}=C_{g}$ läßt sich mit guter Näherung durch eine D eb y e-Funktion darstellen $\left(\Theta_{D}=275\right.$ bis $\left.295^{\circ}\right)$. Die angegebene Beziehung behält ihre Gültigkeit bis $1000^{\circ}$ abs. Die Anharmonizitätskorrektur dürfte danach wenigstens beim Palladium sehr klein sein, da sie sich bis zu diesen Temperaturen nicht bemerkbar macht. Für die Normalentropie des Pd ergibt sich bei $25^{\circ} \mathrm{C}$ ein Wert von 9,04 Clausius.

\section{A. Aufgabenstellung}

1. Die durch eine Messung der spezifischen Wärme zugängliche Atomwärme eines einatomigen Metalls besteht nach unseren gegenwärtigen Kenntnissen aus vier Anteilen:

a) Dem von den Atomschwingungen herrührenden Beitrag, der theoretisch durch die Berechnung des Spektrums des Gitters erfaßt werden kann. Die theoretischen Ansätze liefern die Atomwärme konstanten Volumens $C_{v}$; unter ihnen wird die von Debye gegebene Darstellung wegen ihrer Einfachheit und Klarheit allen übrigen Behandlungsarten meist vorgezogen. Im folgenden legen wir sie ebenfalls zugrunde, zumal Blackman gezeigt hat, daß bei Gittern aus gleichschweren Massenpunkten die Atomwärme als
Funktion eines einzigen Parameters $\Theta_{D}$, der Debyeschen charakteristischen Temperatur, in einem weiten Temperaturbereich recht gut wiedergegeben werden kann.

b) Der Differenz $C_{p}-C_{v}$ der Atomwärmen konstanten Druckes und konstanten Volumens, die rechnerisch ermittelt werden muß, da die calorimetrische Messung stets nur $C_{p}$ liefert. Sie läßt sich bei Zimmertemperatur und darunter genau berücksichtigen und macht einige Prozent von $C_{p}$ aus. So beträgt sie z. B. für Silber bei $20^{\circ} \mathrm{C} 6,084-5,848=0,236 \mathrm{cal} /{ }^{\circ}$, also $3,9 \%$ von $C_{p}$.

c) Einem von den Metallelektronen herrührenden Anteil $C_{e}$, dessen Berechnung für freie Elektronen zuerst Sommerfeld angegeben hat ${ }^{1}$.

1 A. S om mer feld, Z. Physik 47, 1 [1928]. 
Nach ihm ist

$$
\begin{aligned}
C_{e} & =\frac{\pi^{2} n m k}{h^{2}}-\left(\frac{8 \pi V}{3 n N_{L}}\right)^{2 / 3} R T \\
& =3,26 \cdot 10^{-5} V^{2 / 3} n^{1 / 3} T\left[\mathrm{cal} /{ }^{0} \mathrm{~g} \text {-Atom }\right]
\end{aligned}
$$

( $m$ die Elektronenmasse, $V$ das Atomvolumen, $n$ die Zahl der freien Elektronen pro Atom, $N_{L}$ die Loschmidtsche Zahl, $h$ das Plancksche Wirkungsquantum, $k$ die Gaskonstante pro Atom und $R$ die universelle Gaskonstante). Bei einem typischen Metall wie Silber ist die Zahl der freien Elektronen $n_{e}=1$ und das Atomvolumen $V$ in der Größenordnung von $10 \mathrm{ccm}$; man findet dann

$$
C_{e}=1,54 \cdot 10^{-4} \mathrm{~T} \mathrm{cal} / 0 \mathrm{~g} \text {-Atom } .
$$

Danach beträgt die Elektronenwärme bei Zimmertemperatur nur $0,045 \mathrm{cal} /{ }^{\circ}$, d.h. $0,74 \%$ der Atomwärme. Ihr Anteil ist"somit rund 5-mal kleiner als die unter b) erwähnte Korrektur $C_{p}-C_{v}$, und es leuchtet ein, daß sie wegen ihrer Geringfügigkeit nur schwierig zu ermitteln sein wird, wenn wirklich nur die freien Elektronen einen Beitrag liefern.

d) Noch unsicherer ist schließlich eine bei hohen Temperaturen nach Born und Brody zu erwartende Korrektur $C_{a}$, die von der Anharmonizität der Gitterschwingungen herrührt. Die genannten Autoren leiteten dafür ebenfalls einen $T$ proportionalen Anstieg ab, so daß sie von der Elektronenwärme gar nicht ohne weiteres zu trennen ist. Sie kommen ferner zu dem Schluß, daß diese Korrektur meist positiv sein dürfte $^{2}$. Eucken vertritt die entgegengesetzte Auffassung; nach ihm muß die Anharmonizitätskorrektur bei konstantem Gesamtvolumen wegen der mit zunehmender Elongation rasch anwachsenden abstoßenden Kräfte unter allen Umständen negativ sein ${ }^{3}$.

2. Die Möglichkeiten, die interessante Elektronenwärme nachzuweisen, übersehen wir am besten, wenn wir die Grenzfälle hoher und tiefer Temperaturen betrachten.

Bei hohen Temperaturen sollte die von den Gitterschwingungen herrührende Atomwärme schließlich den klassischen Grenzwert von $3 R$ $=5,958 \mathrm{cal} /{ }^{\circ}$ erreichen. Dieser Betrag muß von

2 M. B o r n u. E. B r o d y, Z. Physik 6, 132 [1921].

3 A. E u cken, Lehrbuch d. Chem. Physik, Akadem. Verlagsgesellschaft Leipzig 1944, Bd. II, 2. Tl., S. $672 / 673$. der gemessenen Atomwärme abgezogen und der Rest unter die Anteile $C_{p}-C_{v}, C_{e}$ und $C_{a}$ aufgeteilt werden. Tatsächlich ist aber allen dahin zielenden Bemühungen bis jetzt ein wirklich überzeugender Erfolg versagt geblieben. Wie unsicher dieses Vorgehen ist, ersieht man am besten aus der vortrefflichen Prüfung der Frage durch E u cken ${ }^{4}$. Die Gründe für das unbefriedigende Ergebnis sind in folgenden Tatsachen zu sehen:

a) Die Elektronenwärme erscheint unter allen Umständen als Differenz großer Zahlen, ist also mit sämtlichen Versuchsfehlern unverhältnismäßig belastet;

b) dieser Umstand ist um so mißlicher, als die $C_{p}$-Messungen um so unsicherer werden, je höher die Versuchstemperaturen liegen. Dadurch wird der an sich günstige Einfluß, daß $C_{e}$ linear mit $T$ wächst, zunichte gemacht;

c) ferner fällt auch die mit der Temperatur ansteigende Korrektur $C_{p}-C_{v}$ immer mehr ins Gewicht und läßt sich mangels thermodynamischer Unterlagen bei hohen Temperaturen nicht so genau ermitteln wie bei tiefen;

d) schließlich muß sich die Anharmonizitätskorrektur von $\mathrm{B}$ orn-Brody mit steigender Temperatur irgendwie bemerkbar machen. Wie oben erwähnt, herrscht hier nicht einmal hinsichtlich des Vorzeichens Klarheit, und ihre Absolutberechnung ist vorderhand unmöglich. Wahrscheinlich ist die Korrektur geringfügiger als die Elektronenwärme, und verschiedene Autoren sind heute geneigt, eine beobachtete Uberschreitung des klassischen Grenzwertes von $3 R$ für $C_{v}$ eher auf den Einfluß der Elektronen als auf den der Anharmonizität der Gitterschwingungen zurückzuführen.

3. Bei tiefen Temperaturen trifft man viel günstigere Verhältnisse. Hier verschwinden die Anharmonizitätskorrektur und die Differenz $C_{p}-C_{v}$ vollkommen, so daß neben der Elektronenwärme nur noch der vom Gitter herrührende Anteil auftritt. Es ist nun für den Nachweis von $C_{\boldsymbol{e}}$ ein glücklicher Umstand, daß bei Heliumtemperaturen der Gitteranteil $C_{g}$ nach dem $T^{3}$-Gesetz von Debye, $C_{e}$ jedoch nur linear mit $T$ abnimmt. Man erhält daher

$$
C_{v}=C_{g}+C_{e}=464,5\left(\frac{T}{\Theta_{D}}\right)^{3}+a T
$$

${ }_{4}$ A. Eucken, Handbuch d. Experimentalphys. VIII, 1. Tl., Energie- und Wärmeinhalt, Akadem. Verlagsgesellschaft 1929, S. 214/223. 
Diese Beziehung beschreibt bei einer ganzen Reihe von Metallen zwischen $1^{\circ}$ und etwa $15^{\circ}$ abs die Atomwärme tatsächlich vorzüglich, wie in den Kältelaboratorien von Leiden und Oxford gefunden wurde. Für normale Metalle, $\mathrm{Ag}, \mathrm{Cu}$, Al, wird der Koeffizient des T-Gesetzes dabei durchaus in der nach Gl. (1) zu erwartenden Größenordnung gefunden, wenn man die Zahl der freien Elektronen zu 1 bis 3 annimmt. Dagegen ist bei den Übergangsmetallen $\mathrm{Co}, \mathrm{Ni}, \mathrm{Pt}$ und $\mathrm{Pd}$ der Proportionalitätsfaktor von $T$ etwa eine Zehnerpotenz größer, als man erwartet, wenn die Elektronenwärme nur von den freien Elektronen verursacht wird. So kann nach Pickard zwischen 2,5 und $22^{\circ}$ abs die Atomwärme des Palladiums durch den Ausdruck

$$
{ }_{2,5^{\circ}}^{22^{\circ}} C_{v}=464,5\left(\frac{T}{275}\right)^{3}+31 \cdot 10^{-4} T\left[\mathrm{cal} /{ }^{0} \mathrm{~g} \text {-A tom }\right](3)
$$

wiedergegeben werden ${ }^{5}$. Dabei ist der Zahlenfaktor vor $T$ rund 22-mal größer, als sich aus Gl. (1) bei Wirksamkeit eines einzelnen freien Elektrons errechnet! Bei den ferromagnetischen oder zumindest stark paramagnetischen Metallen sind offenbar die wenigen freien Elektronen überhaupt nicht die Ursache der anomal hohen Elektronenwärme. Sicherlich ist bei ihnen der ursprüngliche Sommerfeldsche Ansatz unzulässig, da er die Wechselwirkung des Elektronengases mit dem Gitter nicht berücksichtigt; darauf kommt es bei diesen Metallen aber gerade an.

4. Wir berichten heute über Messungen der wahren Atomwärme des Palladiums zwischen $12^{\circ}$ abs und Zimmertemperatur. Dieses Metall haben wir mit gutem Grund gewählt. In der ursprünglichen Sommerfeldschen Theorie hängt die in bezug. auf $T$ lineare Beschaffenheit der Elektronenwärme mit der Tatsache einer extrem hohen Entartungstemperatur des Elektronengases von $T_{o} \approx 10^{4}$ Grad zusammen, und das lineare Gesetz muß für Temperaturen $T \ll T_{o}$ gelten, d.h. bis zum Schmelzpunkt der Metalle, mindestens aber bis Zimmertemperatur. Beim Palladium kann nun die Entartungstemperatur keineswegs so hoch liegen, da die Annahme des uneingeschränkten $T$ Gesetzes mit dem bei tiefen Temperaturen ermittelten Faktor zu viel zu großen Werten für die Elektronenwärme bei hohen Temperaturen führt. So müßte nach Gl. (3) bei $1000^{\circ}$ abs die Elek-

5 G. L. P i c k a r d, Nature [London] 138, 123 [1936]. (Das Original war uns nicht zugänglich.) tronenwärme $C_{e}=3,1 \mathrm{cal} /{ }^{\circ}$ betragen, während $C_{p}=7,18 \mathrm{cal} /{ }^{\circ}$ gefunden wird; zieht man noch die Differenz $C_{p}-C_{v}=0,64 \mathrm{cal} /{ }^{\circ}$ in Betracht, so bleibt für den Anteil des Gitters an der Atomwärme der viel zu kleine Wert von $3,44 \mathrm{cal} /{ }^{\circ}$ übrig. Daher muß bei hohen Temperaturen die Elektronenwärme des Palladiums sicher kleiner sein, als aus dem bei Heliumtemperaturen gefundenen Gesetz folgt. Andererseits weist der $C_{v}{ }^{-}$ Wert von $7,18-0,64=6,54$ cal darauf hin, daß der klassische Grenzwert von $3 R=5,958$ cal bei $1000^{\circ}$ abs immer noch viel stärker überschritten wird, als es die ursprüngliche Annahme freier Valenzelektronen fordert. Die Elektronenwärme des Palladiums muß also im ganzen Gebiet beträchtlich sein. Genaue Messungen seiner Atomwärme oberhalb von $12^{\circ}$ abs sollten einen also am ehesten instand. setzen, das Gesetz ausfindig zu machen, dem die Elektronenwärme in einem größeren Temperaturbereich folgt. Dieses Ziel glauben wir auch durch eine phänomenologische Erweiterung des Sommerfeld schen T-Gesetzes erreicht zu haben.

\section{B. Experimentelles}

1. Das Palladium wurde uns von der Firma W. C. H e r a e u s (Hanau) in größter Reinheit freundlichst leihweise zur Verfügung gestellt, wofür wir auch hier bestens danken. Der zylindrische Versuchskörper hatte eine Länge von $36 \mathrm{~mm}$ bei $17,1 \mathrm{~mm}$ Durchmesser; er wurde mit einem $0,05 \mathrm{~mm}$ dicken Konstantandraht zur Zufuhr der elektrischen Heizenergie und einem Bleidraht von $0,07 \mathrm{~mm}$ Stärke zur elektrischen Temperaturmessung bewickelt. Guter Wärmekontakt und elektrische Isolation wurden mit feinstem Seidenpapier und etwas Japanlack erreicht. Die Lackierung ließen wir einige Tage an der Luft trocknen und vollendeten diesen Prozeß durch eine 18 Stdn. währende Erwärmung in einem elektrischen Trokkenschrank, wobei die Temperatur langsam auf $120^{\circ} \mathrm{C}$ gesteigert wurde. Die spezif.Wärme des Lacks wurde durch gesonderte Versuche an analog behandelten Proben im ganzen Temperaturbereich genau bestimmt und ebenso wie die der Heiz- und Bleiwicklung rechnerisch berücksichtigt.

2. Der Versuchskörper wurde in die früher beschriebene Anordnung eingebaut, in der er bis $10^{\circ}$ abs mit festem Wasserstoff abgekühlt werden konnte ${ }^{6}$. Vor und nach jeder Messung wurde der Bleidraht bei $0^{\circ} \mathrm{C}$ auf Konstanz des Widerstandes geprüft und weiterhin durch Eintauchen des ganzen Versuchskörpers in flüssigen Wasserstoff und flüssige Luft geeicht. Bei $20^{\circ}$ abs leiteten wir die Temperatur aus

${ }^{6}$ K. Clusius u. J. Goldmann, Z. physik. Chem. Abt. B 31, 256 [1936]. 
dem Dampfdruck des Bades, bei $81^{\circ}$ abs aus der Tension eines gleichzeitig eintauchenden $\mathrm{O}_{2}$-Dampfdruckthermometers ab. Die Abweichungen der Hat-Werte der Temperaturen des Bleidrahtes von den Soll-Werten der zur Temperaturberechnung benutzten Tabellen waren stets kleiner als $0,15^{\circ}$ und konnten mit der Nernstschen $\alpha$-Regel bequem berücksichtigt werden.

\section{Ergebnisse und Diskussion}

1. Die Ergebnisse für vier verschiedene Meßreihen sind in Tab. 1 verzeichnet.

\begin{tabular}{|c|c|c|c|c|c|}
\hline $\begin{array}{l}\text { Meß- } \\
\text { reihe }\end{array}$ & $T^{0} \mathrm{abs}$ & $C_{p}$ & $\begin{array}{l}\text { Meß- } \\
\text { reihe }\end{array}$ & $T^{0} \mathrm{abs}$ & $C_{p}$ \\
\hline IV/ 1 & 13,70 & 0,1024 & I/ 3 & 88,67 & 3,886 \\
\hline IV/ 6 & 14,84 & 0,1221 & III/ 8 & 89,77 & 3,925 \\
\hline IV /10 & 14,96 & 0,1288 & I/ 4 & 92,38 & 4,018 \\
\hline IV/14 & 16,82 & 0,1537 & III/ 9 & 93,71 & 3,998 \\
\hline IV/ 2 & 16,95 & 0,1509 & I/ 5 & 95,95 & 4,130 \\
\hline IV/18 & 17,33 & 0,1735 & III/10 & 97,35 & 4,216 \\
\hline IV/ 7 & 17,45 & 0,1666 & I/ 6 & 101,63 & 4,311 \\
\hline IV/11 & 17,49 & 0,1673 & I) 7 & 105,82 & 4,435 \\
\hline IV/15 & 19,50 & 0,2192 & I/ 8 & 110,82 & 4,574 \\
\hline IV/ 3 & 19,62 & 0,2188 & I/ 9 & 115,53 & 4,696 \\
\hline IV/ 8 & 19,94 & 0,2367 & $\mathrm{I} / 10$ & 120,16 & 4,792 \\
\hline IV/19 & 20,37 & 0,2518 & $\mathrm{I} / 11$ & 124,73 & 4,878 \\
\hline IV/ 4 & 22,51 & 0,3137 & I/12 & 129,18 & 4,973 \\
\hline IV/ 9 & 22,69 & 0,3203 & $\mathrm{I} / 13$ & 134,49 & 5,072 \\
\hline IV/13 & 24,03 & 0,3716 & I 14 & 138,77 & 5,143 \\
\hline IV'/16 & 25,32 & 0,4298 & $\mathrm{I} / 15$ & 143,67 & 5,213 \\
\hline IV/ 5 & 25,92 & 0,4585 & $\mathrm{I} / 16$ & 149,94 & 5,302 \\
\hline IV/20 & 26,23 & 0,4803 & I/17 & 155,31 & 5,362 \\
\hline IV/17 & 27,83 & 0,5539 & I 18 & 159,99 & 5,432 \\
\hline IV'/21 & 33,20 & 0,8562 & $\mathrm{I} / 19$ & 164,95 & 5,478 \\
\hline IV/22 & 38,57 & 1,199 & $I^{\prime} / 20$ & 170,26 & 5,539 \\
\hline IV/23 & 41,88 & 1,407 & I/21 & 179,53 & 5,633 \\
\hline IV/24 & 44,59 & 1,625 & $I^{\prime} / 22$ & 185,76 & 5,677 \\
\hline IV/25 & 47,90 & 1,826 & I/23 & 191,05 & 5,740 \\
\hline IV'/26 & 50,07 & 2,025 & I/24 & 196,69 & 5,743 \\
\hline III/ 1 & 57,50 & 2,413 & II/ 1 & 201,18 & 5,738 \\
\hline IV/27 & 57,92 & 2,443 & I/25 & 201,87 & 5,766 \\
\hline IV/28 & 61,55 & 2,657 & II/ 2 & 205,34 & 5,820 \\
\hline III/ 2 & 62,31 & 2,692 & $\mathrm{I} / 26$ & 206,95 & 5,821 \\
\hline IV/29 & 65,08 & 2,854 & II/ 3 & 210,52 & 5,888 \\
\hline III/ 3 & 67,38 & 2,992 & II/ 4 & 215,78 & 5,897 \\
\hline III/ 4 & 72,14 & 3,211 & II' 5 & 222,09 & 5.914 \\
\hline IV/30 & 75,10 & 3.350 & II/ 6 & 226,04 & 5,955 \\
\hline III/ 5 & 76,66 & 3,430 & II'/ 7 & 230,15 & 5,970 \\
\hline IV/31 & 78,82 & 3,501 & II/ 8 & 237,31 & 5,973 \\
\hline I/ 1 & 80,70 & 3,532 & II/ 9 & 242,16 & 6,005 \\
\hline III/ 6 & 81,42 & 3,633 & II $/ 10$ & 249,17 & 6,044 \\
\hline IV/32 & 83,61 & 3,695 & II'/11 & 253,02 & 6,081 \\
\hline I/ 1 & 84,43 & 3,707 & II $/ 12$ & 258,67 & 6,100 \\
\hline \multirow[t]{2}{*}{ III/ 7} & 85,34 & 3,762 & II 13 & 263,68 & 6,115 \\
\hline & & & II/14 & 268,44 & 6,133 \\
\hline
\end{tabular}

Tab. 1. Atomwärme von Palladium

(gemessen wurden $92,717 \mathrm{~g}=0,86895 \mathrm{~g}$-Atome; Atomgewicht 106,7).

Meßreihe I am 17.5.44, Bad: flüssige Luft. Meßreihe II am 25.5.44, Bad: $\mathrm{CO}_{2}$-Methylalkohol. Meßreihe III am 8.6.44, Bad: abgepumpte flüssige Luft.

Meßreihe IV am 28.6.44, Bad: fester u. flüssiger $\mathrm{H}_{2}$.
Bei tiefen Temperaturen folgen zwischen 13 und $22^{\circ}$ abs unsere Daten gut der von $\mathrm{Pick}$ ar d in Abschnitt A 3 angegebenen Funktion (3). Diese ist als ausgezogene Kurve in Abb. 1 mit unseren Meßpunkten eingetragen, woraus sich der gute Anschluß der neuen Werte an die Oxforder Beobachtungen ergibt. Immerhin findet man jedoch hier schon eine Andeutung, daß oberhalb von $20^{\circ}$ abs die ausgeglichenen $C_{p}$-Werte etwas tiefer liegen, als die Funktion (3) es erwarten läßt. Zur Veranschaulichung der durch die Elektronenwärme bedingten Anomalie ist noch die D e b y e Funktion für $\Theta=275^{\circ}$ eingezeichnet, die viel

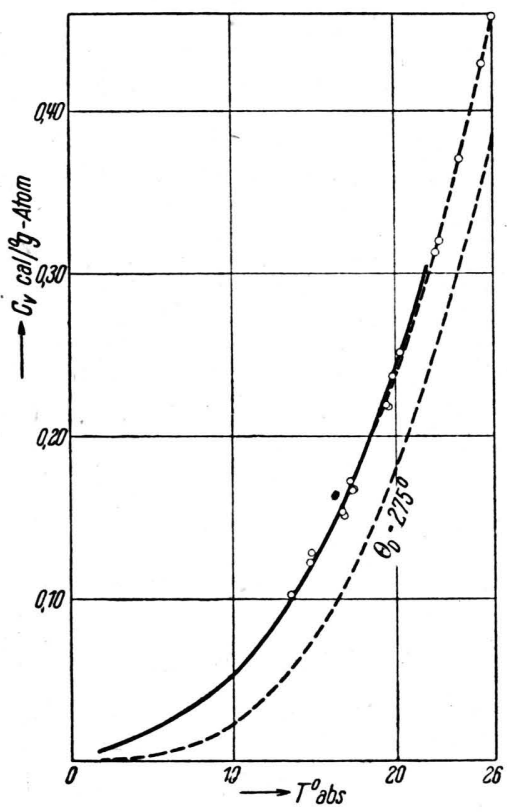

Abb. 1. Atomwärme des Palladiums bei tiefen Temperaturen. Die ausgezogene Kurve entspricht der von $\mathrm{P}$ i c k a r d angegebenen Funktion $C_{v}=464,5$ $(T / 275)^{3}+31 \cdot 10^{-4} T$. Zum Vergleich sind die Meßpunkte dieser Arbeit und die $D$ e b y e-Funktion $C_{v}$ $=D\left(275^{\circ}\right)$ eingetragen. Im Bereich dieses Schaubildes ist $C_{p}$ mit $C_{v}$ gleichzusetzen.

rascher abfällt als die wirklich beobachtete Atomwärme.

Am oberen Ende unseres Meßbereiches erreichen wir die Ordinate für $18^{\circ} \mathrm{C}$ durch eine kleine Extrapolation bei $6,24_{5}$ cal, was recht genau dem von $\mathrm{Jaeger}$ und Diesselhorst ${ }^{7}$ angegebenen Wert $(6,25 \mathrm{cal})$ entspricht. Aus mittleren spezifischen Wärmen haben verschiedene Beobachter

7 W. J a e ger u. H. Die s selhorst, Wiss. Abh. physik. techn. Reichsanst. 3, 269 [1900]. 


\begin{tabular}{|c|c|c|c|c|c|c|c|c|c|c|}
\hline 1 & 2 & 3 & 4 & 5 & 6 & 7 & 8 & 9 & 10 & 11 \\
\hline$T^{0} \mathrm{abs}$ & $C_{p}$ graph. & $C_{p}-C_{v}$ & $C_{v}$ beob. & $\Theta_{C_{v}}$ & $C_{e_{1}}$ & $C_{v}-C_{e_{1}}$ & $\Theta_{\left(C_{v}-C_{e_{1}}\right)}$ & $C_{e}$ & $C_{v}-C$ & $\Theta_{\left(C_{v}-C_{e_{\mathbf{q}}}\right)}$ \\
\hline $\begin{array}{l}20 \\
40 \\
60 \\
80 \\
100 \\
120 \\
140 \\
160 \\
180 \\
200 \\
220 \\
240 \\
260 \\
273,2\end{array}$ & $\begin{array}{l}0,232 \\
1,295 \\
2,566 \\
3,558 \\
4,260 \\
4,790 \\
5,162 \\
5,422 \\
\mathbf{5 , 6 2 6} \\
\mathbf{5 , 7 9 4} \\
5,920 \\
6,010 \\
6,086 \\
6,132\end{array}$ & $\begin{array}{l}\quad \bar{y}, 000_{8} \\
0,005 \\
0,012_{5} \\
0,022_{5} \\
0,034 \\
0,046 \\
0,058_{5} \\
0,070_{5} \\
0,083^{5} \\
0,095_{5} \\
0,107 \\
0,119 \\
0,127\end{array}$ & $\begin{array}{l}0,232 \\
1,294 \\
2,561 \\
3,545_{5} \\
\mathbf{4}_{5}, 237_{5} \\
4,756^{5} \\
5,116 \\
5,363_{5} \\
5,555_{5} \\
\mathbf{5 , 7 1 1} \\
\mathbf{5}, 824_{5} \\
\mathbf{5}, 903 \\
5,967 \\
6,005\end{array}$ & $\begin{array}{l}252 \\
264 \\
270,{ }_{5} \\
273 \\
271 \\
261,{ }_{5} \\
248 \\
234,{ }_{5} \\
214 \\
184 \\
147 \\
101,{ }_{5} \\
-\end{array}$ & $\begin{array}{l}0,062 \\
0,124 \\
0,186 \\
0,248 \\
0,310 \\
0,372 \\
0,434 \\
0,496 \\
0,558 \\
0,620 \\
0,682 \\
0,744 \\
0,806 \\
0,846\end{array}$ & $\begin{array}{l}0,170 \\
1,170 \\
2,375 \\
3,297_{5} \\
3,927^{5} \\
4,384^{5} \\
4,682 \\
4,867_{5} \\
4,997_{5} \\
5,091^{5} \\
5,142_{5} \\
5,159 \\
5,161 \\
5,159\end{array}$ & $\begin{array}{l}279 \\
276 \\
285 \\
294 \\
302 \\
307 \\
316 \\
329 \\
344 \\
361 \\
383 \\
413 \\
447 \\
471\end{array}$ & $\begin{array}{l}0,050 \\
0,090 \\
0,129 \\
0,164 \\
0,198 \\
0,231 \\
0,261 \\
0,289 \\
0,313 \\
0,334 \\
0,353 \\
0,369 \\
0,382 \\
0,391\end{array}$ & $\begin{array}{l}0,182 \\
1,204 \\
2,432 \\
3,381 \\
4,039_{5} \\
4,525 \\
4,855 \\
5,074_{5} \\
5,242_{5} \\
5,377 \\
5,471_{5} \\
5,534 \\
5,585 \\
5,614\end{array}$ & $\begin{array}{l}273 \\
273 \\
281 \\
286,{ }_{5} \\
291 \\
290 \\
290 \\
292 \\
292 \\
289 \\
289 \\
293 \\
297 \\
299\end{array}$ \\
\hline
\end{tabular}

Tab. 2. Atomwärme des Palladiums und ihre Aufteilung zum Nachweis der Elektronenwärme zwischen $20^{\circ}$ und $273,2^{\circ}$ abs.

folgende Interpolationsformeln abgeleitet:

$\mathrm{Behn}^{8}$ :

$$
C_{p}=6,199+3,457 \cdot 10^{-3} t-2,73 \cdot 10^{-5} t^{2},
$$

Jäger und Veenstra ${ }^{9}$ :

${ }_{0^{0}}^{1500^{\circ}} C_{p}=6,229+1,286 \cdot 10^{-3} t+2,75 \cdot 10^{-8} t^{2},(4 \mathrm{~b})$

Holzmann ${ }^{10}$ :

$$
C_{p}=6,232+1,691_{5} \cdot 10^{-3} t .
$$

Aus diesen Beziehungen folgen für $18^{\circ} \mathrm{C}$ die Werte $6,25_{2} \mathrm{cal}^{\circ}{ }^{\circ}$ (B e h n ), 6,25 $\mathrm{cal}^{\circ}$ (J a e g e r u. Veenstra) und $6,26_{2}$ cal $^{\circ}$ (Holzmann). Der Anschluß unserer Messungen an frühere Beobachter ist also auch bei Zimmertemperatur gut.

Aus den Daten der Tab. 1 ergibt sich graphisch die Entropie des Palladiums bei $25^{\circ} \mathrm{C}$ zu 9,04 Clausius.

2. Da unsere Meßpunkte oberhalb $20^{\circ}$ abs mit wenigen Ausnahmen auf 1 bis $2 \%$ genau auf einem glatten Kurvenzug liegen, benutzten wir für die folgenden Betrachtungen von $10 \mathrm{zu} 10^{\circ}$ interpolierte Werte, die auszugsweise in Tab. 2 zusammengestellt sind. Die Differenz $C_{p}^{\prime}-C_{v}$ wurde durch die Beziehung

$$
C_{p}-C_{v}=A T C_{p}^{2}=1,24 \cdot 10^{-5} T C_{p}^{2}
$$

berücksichtigt. Der numerische Wert von $A$ ergab sich aus der exakten Formel ${ }^{11}$

$$
C_{p} / C_{v}=1+a \gamma T
$$

${ }^{8}$ U. B e h n, Ann. Physik 66, 237 [1898].

- F.M. J a e g e r u. W. A. Ve e n s tra, Kon. Akad. Wetensch. Amsterdam, Proc. 37, 280 [1934]. bei $20^{\circ}$ mit den Werten $\gamma=2,23$ und $\alpha=34,5 \cdot 10^{-6}$, dem Ausdehnungskoeffizienten.

3. Die so erhaltenen $C_{v}$-Werte wurden nach drei verschiedenen Verfahren unter Berücksichtigung der Tatsache analysiert, daß es unterhalb der Zimmertemperatur viel aufschlußreicher ist, den Verlauf der $\Theta_{D}$-Werte als den der $C_{v}$-Kurve zu betrachten. Dabei wurde zunächst angenommen, daß in dem von uns untersuchten Temperaturbereich die Born-Brodysche Korrektur wegfällt, so daß die $C_{v}$-Werte außer einem von den Elektronen herrührenden Anteil $C_{e}$ nur noch die von den Gitterschwingungen hervorgerufene Atomwärme enthalten. Bei voller Gültigkeit der D e by e schen Theorie für das Gitter und beim Fehlen einer Elektronenwärme muß der aus $C_{v}$ berechnete $\Theta_{D}$-Wert über den gesamten Temperaturbereich konstant sein.

I. Es wurde zunächst untersucht, wie weit dies für die Atomwärme $C_{v}$ selbst zutrifft, wie weit sie sich also durch eine Deb y e-Funktion allein darstellen läßt:

$$
\Theta_{D}=D\left(C_{v}\right) .
$$

Die aus $C_{v}$ tabellarisch für $\Theta_{D}$ gefundenen Werte sind in Tab. 2, Spalte 5, und auf Abb. 2 durch die ausgezogene Kurve I als Funktion von $T$ wiedergegeben. Von einer Konstanz des $\Theta_{D}$-Wertes kann keine Rede sein. Für das folgende ist $\mathrm{zu}$ beachten, daß ein zu kleiner $\Theta_{D^{-}}$-Wert einer $z u$ großen

${ }^{10} \mathrm{H}$. H o l z mann, S i e b e r t - Festschrift, Hanau 1931.

11 Zahlenwerte nach E. Grü n e is e n, Handbuch d. Physik X, S. 28. 
Atomwärme entspricht und umgekehrt. Der steile Abfall von $\Theta_{D}$ bei tiefen Temperaturen, bei denen sich die zusätzliche Elektronenwärme am deutlichsten bemerkbar macht, veranschaulicht diese Tatsache. Zwischen 30 und $120^{\circ}$ abs durchläuft $\Theta_{D}$ ein breites Maximum, fällt aber dann erst langsam und schließlich rasch auf verschwindende Werte ab. Das Maximum liegt in dem Gebiet, wo die $C_{v}$-T-Kurve ihren Wendepunkt durchlaufen hat und noch steil ansteigt; hier ist $\Theta_{D}$ gegen eine Variation von $C_{v}$ am unempfindlichsten. Bei höheren Temperaturen wird jedoch $\Theta_{P}$ in dem Maße, wie sich die $C_{v}$-Kurve dem Grenzwert $3 R=5,958 \mathrm{cal} /{ }^{\circ}$ nähert, außerordentlich stark durch geringe zusätzliche Wärmeeffekte - hier offensichtlich die Elektronenwärme - beeinflußt, und zwar herabgedrückt. Außer den Ferromagnetica $\mathrm{Fe}, \mathrm{Co}$, Ni ist bisher wohl kaum ein Metall bekannt, das durch einen absonderlichen Verlauf der $\Theta_{D}$-T-Kurve die Anwesenheit der Elektronenwärme im gesamten Temperaturbereich so deutlich verrät wie das Palladium.

II. Welcher Temperaturfunktion folgt aber diese Elektronenwärme? Untersuchen wir probeweise an zweiter Stelle den $\Theta_{D}-T$-Verlauf, wenn die Elektronenwärme $C_{e_{1}}$ mit der bei Heliumund Wasserstofftemperaturen gültigen Formel bis Zimmertemperatur in Rechnung gestellt wird.

$$
\Theta_{D}=D\left(C_{v}-31 \cdot 10^{-4} T\right)
$$

Wir wissen zwar, daß diese Darstellung bei $1000^{\circ}$ abs bestimmt versagt (s. A 4), immerhin können wir aber so feststellen, wann und wo die Abweichungen vom linearen Gesetz beginnen. Die erhaltenen $\Theta_{B}$-Werte stehen in Tab. 2, Spalte 8, und sind in Abb. 2 durch die punktierte Kurve II veranschaulicht. Man erkennt, daß $\Theta_{D}$ bei tiefen Temperaturen bis etwa $40^{\circ}$ abs konstant ist, um dann monoton stark anzusteigen. Ein solcher Verlauf der $\Theta_{D}$-T-Kurve wird bei einem Element „,ohne Gruppenbildung“ niemals beobachtet. Physikalisch würde er bedeuten, daß die Grenzfrequenz mit steigender Temperatur schneller wird, wozu kein ersichtlicher Grund vorliegt. Da dieser $\Theta_{D^{-}}$-Verlauf $\mathrm{zu}$ kleinen Atomwärmen entspricht, wird er offensichtlich dadurch vorgetäuscht, daß die Elektronenwärme nach Gl. (6b) $\mathrm{zu}$ hoch angesetzt ist, und daß die Abweichungen vom linearen Gesetz - und zwar Unterschreitungen - bereits dicht oberhalb von Wasserstofftemperaturen beginnen. Tatsächlich dürfte das schon oben erwähnte, auf Abb. 1 bemerkbare $\mathrm{Zu}$ rückbleiben der ausgeglichenen $C_{v}$-Werte gegenüber der Pickardschen Kurve oberhalb $20^{\circ}$ abs auf das beginnende Versagen des linearen Gesetzes für $C_{e}$ zurückzuführen sein.

III. Um zu einer rationalen Darstellung der Verhältnisse zu gelangen, erinnerten wir uns, daß M o t t zuerst auf eine Proportionalität zwischen $C_{e}$ und $\chi_{e} T$ bei freien Elektronen hingewiesen hat, wobei $\chi_{e}$ die von den Elektronen herrührende paramagnetische Atomsuszeptibilität bedeutet ${ }^{12}$.

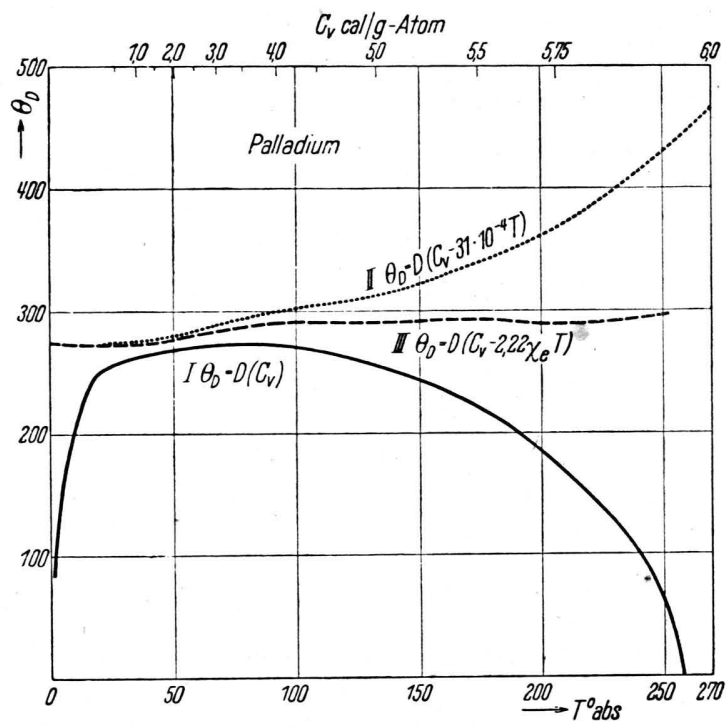

Abb. 2. Verlauf der $\Theta_{D}$-Werte des Palladiums für verschiedene Berechnungsarten. Näheres siehe Text.

Wird mit $\chi_{D}$ die diamagnetische Atomsuszeptibilität der positiven Metallionen und mit $\chi_{A}$ die experimentell gemessene Atomsuszeptibilität bezeichnet, so gilt

$$
\chi_{A}=\chi_{e}-\left|\chi_{D}\right|
$$

Nach M o t $t$ ergibt sich bei tiefen Temperaturen für das Verhältnis

$$
C_{e} / \chi_{e} T=\pi^{2} k^{2} / 3 \mu^{2}
$$

( $\mu$ das Bohrsche Magneton). Für den Fall eines Paramagnetismus „kollektiver" Elektronen tritt auf die rechte Seite von Gl. (8) noch ein Faktor, der die Wechselwirkung zwischen Elektronen und Gitter berücksichtigt, sich aber numerisch nicht ohne weiteres feststellen läßt ${ }^{13}$. Wir

12 M o t t, Proc. physic. Soc. 47, 571 [1935],

13 E. C. St o n e r, Proc. Roy. Soc. [London] Ser. A 154, 656 [1936]. 
sind nun versuchsweise so vorgegangen, daß wir 1. für den gesamten Temperaturbereich die Proportionalität zwischen $C_{e}$ und $\chi_{e} T$ angenommen und 2. den Proportionalitätsfaktor in der Weise normiert haben, daß die resultierende Formel bei tiefsten Temperaturen in das lineare Gesetz einmündet. Wir erhalten so unter Berücksichtigung von (3) die Beziehung

$$
C_{e}=\frac{31 \cdot 10^{-4} \chi_{e} T}{\chi_{e_{0}}} ;
$$

in dieser bedeutet $\chi_{e}$ die temperaturabhängige Elektronensuszeptibilität und $\chi_{\boldsymbol{e}_{0}}$ deren Wert bei den tiefsten Temperaturen. Für $\chi_{A}$ liegen Messun-

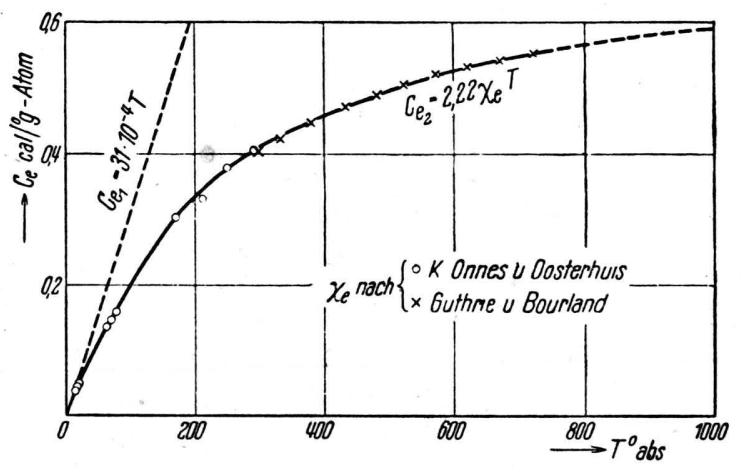

Abb. 3. Verlauf der Elektronenwärme $C_{e}$ des Palladiums.

gen von $\mathrm{K}$. Onnes und $\mathrm{O}$ os te $\mathrm{r} \mathrm{h}$ u is zwischen $14^{\circ}$ abs und Zimmertemperatur ${ }^{14}$, anschließend bis $450^{\circ} \mathrm{C}$ solche von $\mathrm{Guthrie}$ und $\mathrm{B}$ ou r l a n d vor ${ }^{15}$, deren Ergebnisse gut zu den holländischen Werten passen. Durch Extrapolation der gegen $T$ aufgetragenen $1 / \chi_{\Lambda}$-Werte, die freilich mit einer gewissen Unsicherheit behaftet ist, finden wir unter Berücksichtigung der temperaturunabhängigen diamagnetischen Atomsuszeptibilität ${ }^{16}$ des Palladiums von $\chi_{D}=-58 \cdot 10^{-6}$ schließlich $\chi_{e_{0}}$ zu $1390 \cdot 10^{-6}$. Wir erhalten dann aus Gl. (9)

$$
C_{e_{2}}=2,22 \cdot \chi_{e} T[\mathrm{cal} / 0]
$$

für die „kollektive“ Elektronenwärme. Diese Funktion ist auf Abb. 3 an Hand der Meßpunkte für die Suszeptibilität eingetragen. $\mathrm{Zu}$ der geringen Extrapolation oberhalb von $700^{\circ}$ abs wurde

14 H. K. Onnes u. E. O osterhuis, Commun. physic. Lab. Univ. Leiden 139 e; Kon. Akad. Wetensch. Amsterdam, Proc. 1914, 1033. die C u ri e-Wei $ß$ sche Beziehung

$$
\chi_{e}=\frac{C^{\prime}}{T-\Theta^{\prime}}-\chi_{D}
$$

benutzt $\left(C^{\prime}=3,044 \cdot 10^{-3}\right.$ und $\left.\Theta^{\prime}=-227^{\circ}\right)$. Vom Nullpunkt ausgehend ist noch gestrichelt die von P i cka rd bei Helium-Wasserstofftemperaturen gefundene, linear mit $T$ ansteigende Elektronenwärme $C_{e_{1}}$ vergleichsweise eingezeichnet.

Die so festgelegte Elektronenwärme $C_{e_{2}}$ befriedigt nun die experimentellen Daten für die Atomwärme überraschend gut. In Tab. 2 sind in Spalte 9 die aus Abb. 3 abgelesenen $C_{\boldsymbol{e}_{\mathbf{z}}}$-Werte, in Spalte 10 die $C_{v}-C_{e_{2}}$-Werte und in der letzten Spalte die daraus resultierenden $\Theta_{D}$-Werte angegeben. Letztere sind ferner als Kurve III in Abb. 2 eingetragen. Man erkennt sofort, daß bis auf einen geringfügigen Gang und kleinere Unregelmäßigkeiten die so ermittelten $\Theta_{D^{-}}$Werte nunmehr nahezu konstant sind und jedenfalls systematische Abweichungen nach hohen Temperaturen zu nicht mehr zeigen. Man darf nicht übersehen, daß man sich bei $0^{\circ} \mathrm{C}$ in der Nähe des Äquipartitionswertes $3 R$ befindet, und hier der $\Theta_{D^{-}}$ Wert gegen geringe Fehler von $C_{v}$ sehr empfindlich ist; eine Abweichung von $0,1 \%$ in $C_{v}$ spiegelt sich in einer Abweichung von fast $1 \%$ von $\Theta_{D}$ wider. Das bedeutet aber, daß $C_{e}$ bei dieser Temperatur auf $1,5 \%$ genau festgelegt sein muß.

Die gefundene gute Übereinstimmung ermutigte uns, zu untersuchen, ob auch oberhalb von Zimmertemperatur die aufgestellten Beziehungen für $C_{p}, C_{p}-C_{v}$ und $C_{e_{2}}$ noch anwendbar sind. Wie diese Prüfung zwischen 300 und $1000^{\circ}$ abs ausfällt, geht aus Tab. 3 hervor. Für die $C_{p}$-Werte wurde die oben von $\mathrm{J}$ äge $\mathrm{r}$ und Veen stra angegebene Funktion benutzt (s.C1). Die letzte Spalte zeigt, daß die so ermittelten Werte für den Anteil des Gitters an der Atomwärme sich sehr allmählich dem Grenzwert von 5,958 cal nähern und ihn erst bei etwa $1000^{\circ}$ erreichen. Auch dieses Ergebnis muß als befriedigend bezeichnet werden. Eine Verfolgung zu noch höheren Temperaturen haben wir jedoch nicht vorgenommen, wohl bewußt, daß der Gültigkeitsbereich aller verwendeten Beziehungen - insbesondere auch der immer mehr "ins Gewicht fallenden $C_{p}-C_{v^{-}}$

15 A. N. Guthrie u. L.T.Bourland, Physic. Rev. 37, 303 [1931].

16 Siehe z. B. E.St o ner, Magnetism and matter, S. 516/517, Methuen \& Co. [1934]. 